\title{
'Isolated' pulmonary valve stenosis as part of more widespread cardiovascular disease
}

\author{
Luis Beçu, Jane Somerville, and Amelia Gallo \\ From the Department of Pathology, Hospital de Ninos, Gallo 1330, Buenos Aires; and the Paedriatric \\ and Adolescent Unit, National Heart Hospital, London
}

In 25 patients aged 6 days to 9 years presenting as 'isolated' pulmonary valve stenosis, histology of the myocardium of right and left ventricles, coronary arteries, and ascending aorta has shown abnormality in one or all these areas. Myocardial necrosis, old and recent, unrelated to coronary occlusion was frequent. Myocardial 'dysplasia' involving both zentricles, and resembling hypertrophic cardiomyopathy (HOCM, ASH) was found in 10 and a relation of this to myocardial injury in the fetus is postulated. Varying degrees of coronary occlusion were frequently seen in both right and left coronary arteries. The histology of the ascending aorta was abnormal showing 'higgledy-piggledy' disorder of smooth muscle components in 12 (48\%).

In a different series of 53 patients who had pulmonary valvotomy for apparent 'isolated' pulmonary valve stenosis there were 14 with clinical evidence of left ventricular abnormality consistent with the pathological changes described, 2 with the same aortic histological changes, and 2 with macroscopical left ventricular hypertrophy. Two of them developed classical hypertrophic cardiomyopathy years later.

It is suggested that when pulmonary valve stenosis presents with a thick tricuspid poorly mobile valve, particularly in infants or in patients with evidence of other congenital stigmata, it may be part of a more widespread cardiovascular abnormality. This should be recognized and considered in the evaluation of surgical patients and late survivors who may show unexpected clinical features.

Isolated pulmonary valve stenosis with intact ventricular septum is an example of a simple congenital obstruction to blood flow. It has been assumed that surgical opening of the narrow pulmonary valve solves the patient's problem. This straightforward concept has remained unchallenged over the years.

Responsibility for this restricted view may lie on the shoulders of the pathologists. What appeared to be a consistently dome-shaped valvar obstruction with predictable functional muscular hypertrophy proximal to it has not stimulated further research into the true nature and extent of the disease. Specimens have been described routinely with perfunctory mention of the diameter of the valve orifice, the number of cusps, and the thickness of the right ventricular wall and then, more often than not, destroyed.

The purpose of this paper is to report a new aspect of the disease hitherto described as simple pulmonary valve stenosis. Fresh evidence suggests Received 20 October 1975. that in some patients the fusion of the pulmonary valve cusps should be looked upon as the end result of a disease of the fetus which is not necessarily limited to the pulmonary valve, since striking changes may also be present in myocardial architecture in one or both ventricles, in the coronary arteries, and in the aortic wall. Before valvotomy, the dominant manifestations of right ventricular outflow obstruction may obscure the functional significance of these other pathological changes. After apparently successful surgical relief of the obstruction, one or some of the other features of the underlying disease may become obvious though this does not happen in every patient.

Long-term follow-up of survivors after pulmonary valvotomy and retrospective scrutiny of the postoperative course of patients under 3 years of age with severe pulmonary stenosis have revealed some unexpected clinical features which also suggest that the disease may not be limited to the pulmonary valve in every patient. 


\section{Subjects and methods}

Twenty-five specimens preserved in formalin from patients with 'isolated' pulmonary valve stenosis were available for study in the pathology department of the Children's Hospital, Buenos Aires. The ages ranged from 6 days to 9 years-9 were over 1 year. Cases of pulmonary valve stenosis in young infants with a small tricuspid ring diameter were excluded arbitrarily since the clinical picture is different and it is apparent that in them the disease is not confined to the pulmonary valve. By definition none had associated ventricular septal defects or other obvious structural congenital cardiovascular abnormalities. A patent foramen ovale, flaccid or distended, was the rule, and none had a true atrial septal defect; one patient had a closed intact atrial septum.

Eighteen patients with clinical evidence of severe pulmonary stenosis had been operated upon and died hours to 6 days after operation. The other 7 patients died before operation, a few hours or days after admission for undetermined causes, and after cardiac catheterization in one instance. There were no stigmata of rubella outside the cardiovascular system such as cataracts, hepatitis, or diffuse purpura, but rubella as the aetiology cannot be excluded in some because of incomplete clinical data.

Seven to 15 random blocks were taken from the myocardium of both ventricles. Further blocks of tissue were taken containing the main coronary arteries and a sizeable portion of the adjacent muscle, and transverse tissue blocks with the ascending trunk of both the aorta and the pulmonary artery in one section. Paraffin-embedded sections were stained routinely with P.A.S.-haematoxylin, Gomori's aldehyde fuchsin stain for elastic tissue and Masson's trichrome stain for muscle and connective tissue. In selected cases, stains for calcium were used, and also the tetrazolium technique to show necrotic muscle. All slides were evaluated by 2 observers unaware of the clinical details.

Smooth muscle changes in the coronary arteries were graded into 3 categories (Table 1). They were considered to be of 'moderate' severity when more than half the random sections showed normal arteries and the others had the segmental musculoelastic dysplasia described by Neufeld (1974). Cases were classified as having 'widespread' coronary pathology when these moderate changes were present in all random sections, yet only rarely encroaching seriously upon the lumen. 'Severe' coronary obstructive lesions were those that reduced the lumen of subepicardial arteries by 75 per cent or more; cases were included in this category if this degree of obstruction was present in one or
TABLE 1 Summary of pathological changes in 25 patients with 'isolated' pulmonary valve stenosis

\begin{tabular}{|c|c|}
\hline (a) Coronary arteries $\begin{array}{l}\text { Obstructive } \\
\text { Unusually dilated }\end{array}$ & $\left\{\begin{array}{l}\text { moderate } \\
\text { widespread } \\
\text { severe }\end{array}\right.$ \\
\hline (b) Myocardium & $\left\{\begin{array}{l}\text { necrosis } \\
\text { 'dysplasia' } \\
\text { normal }\end{array}\right.$ \\
\hline $\begin{array}{l}\text { Left ventricular pathology } \\
\qquad \text { (a) Coronary arteries Obstructive }\end{array}$ & $\left\{\begin{array}{l}\text { widespread } \\
\text { severe } \\
\text { normal }\end{array}\right.$ \\
\hline (b) Myocardium & $\left\{\begin{array}{l}\text { necrosis } \\
\text { 'dysplasia' } \\
\text { normal }\end{array}\right.$ \\
\hline Aortic wall pathology & $\begin{array}{l}\text { abnormal } \\
\text { normal }\end{array}$ \\
\hline
\end{tabular}

more sections irrespective of what was found in other areas.

Areas of myocardial cell death (necrosis) were considered to be significant when more than onehalf of a mid-power $(\times 40)$ microscopical field was involved. 'Recent' myocardial cell necrosis was diagnosed when the obvious nuclear and cytoplasmic changes were associated with a minimal or no inflammatory reaction. 'Healed myocardial necrosis' was recognized as an area of replacement fibrosis, usually quite dense and uniform but rarely extending beyond a low-power $(\times 10)$ microscopical field.

\section{Results}

\section{Histopathological study}

Eighteen of the pulmonary valves had been opened at surgery so that verification of the residual diameter of the stenotic orifice was inexact. Three were noted to have an eccentric orifice on the original surgical protocol. In 19 specimens the valves were thickened by oedematous stiff connective tissue and it was difficult to distinguish the line of fusion between cusps, but in all there was a clear raphe at the attachment of the valvar tissue to the pulmonary wall indicating the past existence of a tricuspid primordia in every specimen (Fig. 1). There was no example of a stenosed bicuspid pulmonary valve. In the other 6 patients the valvar tissue was thinner, pliable, and tricuspid, almost transluscent. Four of these were aged 2, 4, 7, and 9 


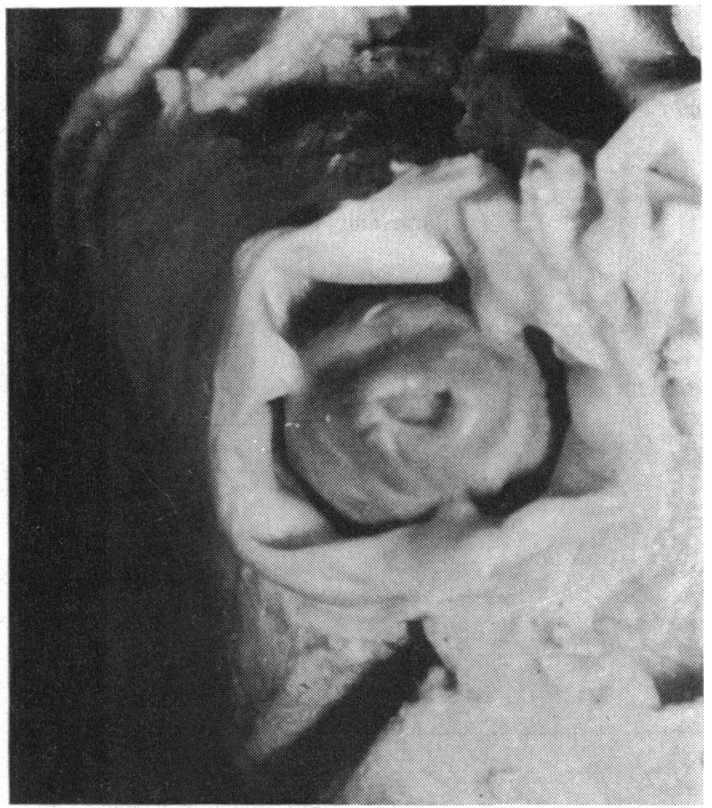

FIG. 1 Typical thickened tricuspid pulmonary valve stenosis from child aged 4 months who died before surgery. Child had extensive areas of myocardial necrosis in both ventricles and occlusive coronary artery changes of moderate severity in the right ventricle.

years and the other 2 were less than 1 year of age.

The histological findings are summarized in Table 1 . The coronary arteries in the right ventricle were abnormal in every patient. The changes were considered to be of moderate degree in 6 and were widespread in a further 10 . Seven patients had severely obstructive lesions (Fig. 2) and all of them were less than 6 months of age. In 2 patients, aged 6 and 8 months, the main and also the intramyocardial arteries were unusually dilated, almost double the normal diameter, but neither showed abnormality of the muscular media or the intima. In both, the histology of the ascending aorta was abnormal showing the typical 'higgledy-piggledy' disorder to be discussed later.

The left ventricular coronary arteries showed no significant abnormality in 15 patients and widespread changes in the subepicardial coronary arteries in 7, all of whom had similar lesions in their right coronary arteries. Severe obstructive lesions were found in coronary arteries on the right in 7 and were also present on the left in 3 of this group.

Histological evidence of recent and/or healed myocardial necrosis was found in the right ventricular wall of 12 patients, in 3 of whom it was also present in the left ventricular wall. As is characteristic in infants, necrotic areas were frequently small and spotty, sometimes coalescing to account for 50 per cent of a low-power field. In addition to the usual discrete inflammatory and macrophage reaction around areas of relatively recent necrosis, mitosis in myofibres was noted in patients under 6 months of age and occasionally pigment deposition was a prominent feature. Since random sections were used, no attempt could be made to quantify the total amount of necrosed myocardium which in 4 specimens was impressive. Even though dating of reparative stages in infancy is imprecise, it was considered that in all these 12 patients myocardial cell necrosis had occurred, at least in part, before operation. In 4 of the 7 patients dying before operation, recent necrosis was a prominent feature. Healed myocardial necrosis was seen as scattered areas of fibrosis, sometimes quite extensive and localized and often interspersed with strands of viable muscle. Characteristically these scars did not follow coronary artery distribution patterns and were not preferentially subendocardial. In 2 of these patients some areas of scarring were associated with the appearances of 'nodular' proliferation of muscle giving rise to a picture curiously similar to some forms of post-necrotic hepatic cirrhosis.

In 10 patients extensive areas of morphological disarray and malalignment of myofibres (Fig. 3) referred to in this paper as myocardial 'dysplasia' were found in sections from the right ventricle. In 7 this change was present in the left ventricle: 4 of these showed the same appearances in sections from right ventricular myocardium. In these areas of 'dysplasia', muscle bundles and single fibres coursed in different directions in a disorderly fashion, forming occasional whorls often at right angles to one another (Fig. 3a and b). The extent and distribution of areas of myocardial 'dysplasia' could not be fully assessed because of the random sampling of ventricular tissue, but it was present in both ventricular septum and the muscle of the free wall and on occasions could be seen by the naked eye (Fig. 3c). None showed localized asymmetrical septal hypertrophy.

Histological examination of the wall of the macroscopically normal ascending aorta showed a disarray and malalignment of its smooth muscle components in 12 patients (Fig. 4), 2 of whom had widely dilated coronary arteries, 3 had moderate coronary changes, 2 were severe, and 5 were widespread. The aortic changes were most obvious with elastic tissue stains since the elastic laminae follow the spatial distribution of the smooth muscle cytoplasm (Fig. 4). Typically the elastic fibres were 

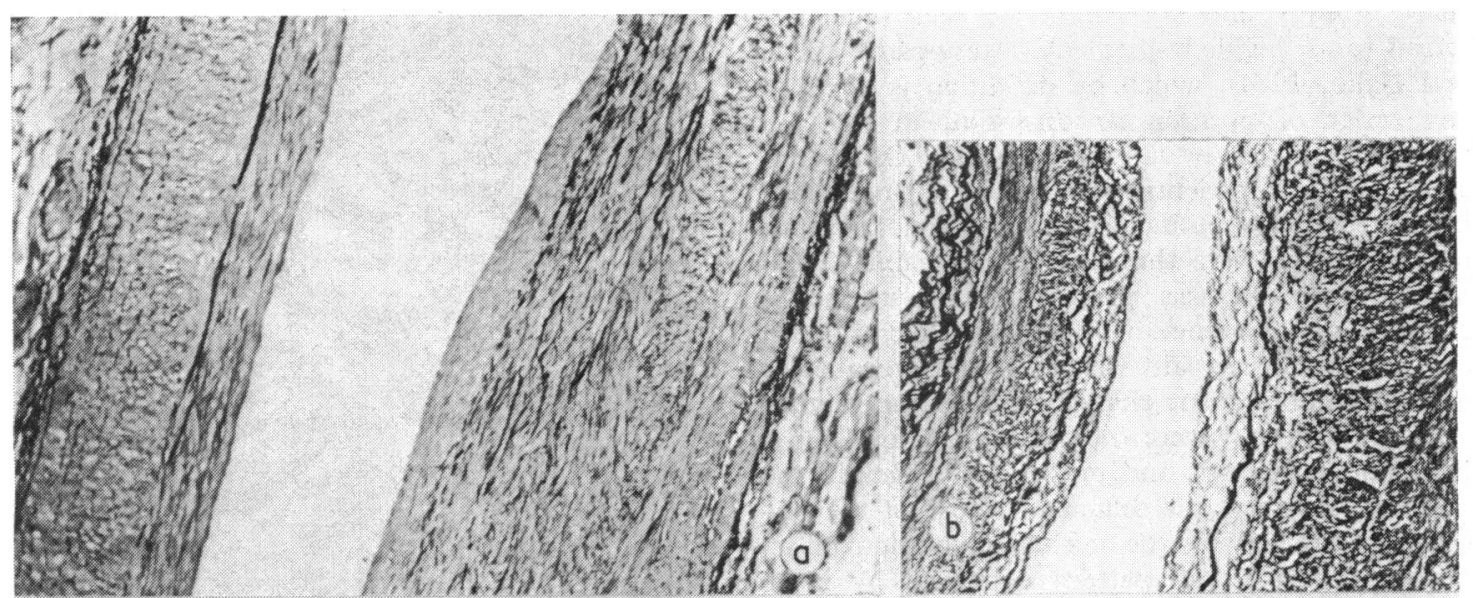
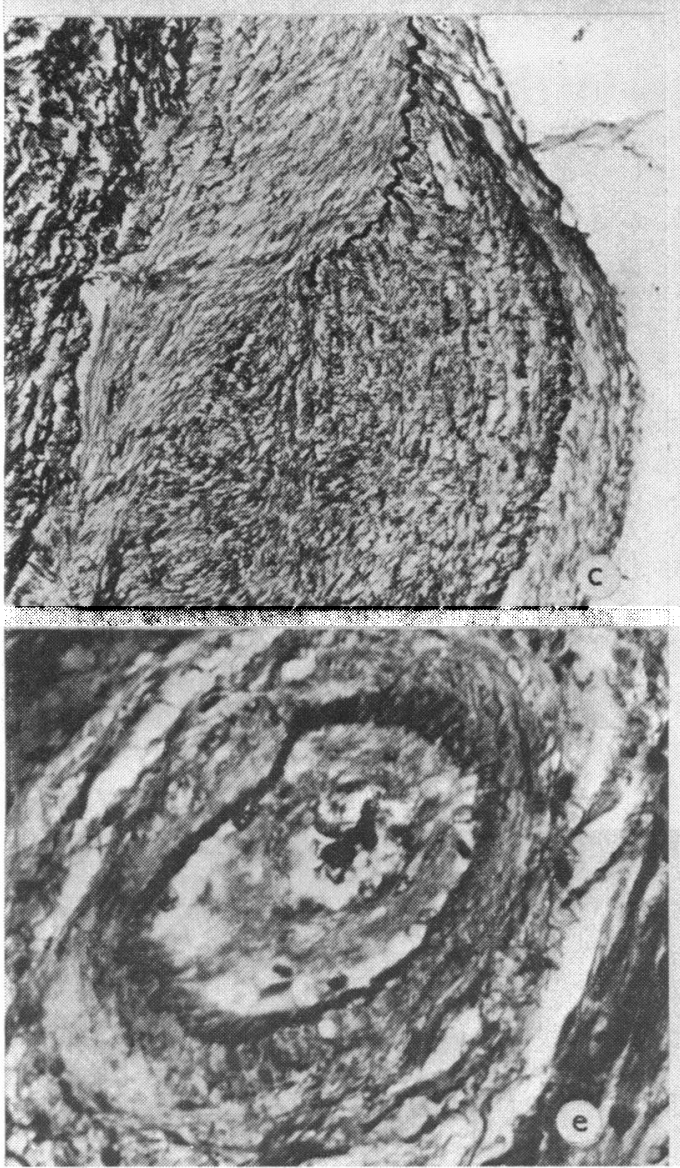

FIG. 2 Coronary arterial pathology in 'isolated' pulmonary valve stenosis. Sections stained with Gomori's aldehyde fuchsin stain for elastic tissue. (a) Longitudinal section of a right ventricular coronary artery from 6-day-old baby. The media to the right is twice as thick as the opposite wall and

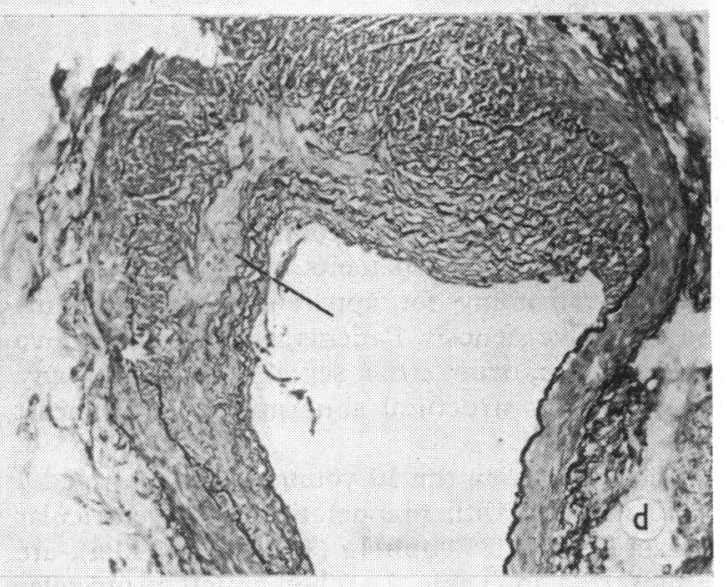

shows much disarray of smooth muscle and elastic fibres $(\times 10)$. Whereas the internal elastic lamina in the left segment is well formed and straight that on the right is not clearcut but it is ill defined and not continuous. There is considerable intimal proliferation on both. (b) Left ventricular intramyocardial coronary artery from a child of 1 year. The medial smooth muscle disarray is prominent, as is the considerable thickening of the segment to the right of this longitudinal section $(\times 10)$. (c) Higher power of a left ventricular coronary artery from a 4-month-old child. The normal media above is replaced by the hamartomatous proliferation of smooth muscle with interruption of the original internal elastic lamina $(\times 40)$. (d) In this coronary artery a segment of the media is totally replaced by a hamartomatous proliferation of smooth muscle, with disappearance of the internal elastic lamina. The arrow indicates an early accumulation of foamy myocytes. The patient was 8 days of age $(\times 10)$. (e) An intramyocardial artery in the left ventricle of a 2-month-old patient showing almost total occlusion $(\times 40)$. 
short, stumpy, and not parallel to each other, referred to as 'higgledy-piggledy' arteriopathy (Beçu and Gallo, 1974), which by definition is 'without any order of position or direction, in jumbled confusion' (Shorter Oxford English Dictionary, 1944). Eleven patients with aortic changes had thickened lumpy pulmonary valves (Fig. 1) and in one the cusps were thin and more pliable. Myocardial dysplasia was present in 7 with aortic changes, and evidence of myocardial necrosis, old and recent, was equally distributed between those with or without aortic changes. In this series other systemic arteries were not available for study. Evaluation of elastic and muscular changes in the pulmonary artery was difficult because of the uncertainty created by the normal involution of its initial aorta-like wall pattern enhanced by poststenotic dilatation.

\section{Possible clinical correlations}

In an attempt to see if there were clinical features which correlated with the above pathological findings, the postoperative course was studied in a different series of 53 patients who had open pulmonary valvotomy for apparently 'isolated' pulmonary valve stenosis. Patients with tricuspid valve abnormalities, true atrial septal defect, and any other obvious structural abnormality in the heart were excluded.

The findings on the 10 youngest patients aged 7 to 30 months, with preoperative right ventricular pressures 95 to $250 \mathrm{mmHg}$ ( 12.6 to $33.3 \mathrm{kPa}$ ), are summarized in Table 2 . Whether left ventricular disease can account for the low output state and death in 3 is uncertain; one patient at necropsy had a large left ventricle (Fig. 5), but there is no histological information available in any nor are the other 2 specimens available to us for inspection. Presumably left ventricular disease was present in the

FIG. 3 Myocardial 'dysplasia' in patients with 'isolated' pulmonary valve stenosis. (a) $\times 8$ (b) $\times 31$. Section from the left ventricle of a child aged 2 years stained with Masson's trichrome. This illustrates the characteristic and striking malalignment and disarray of myocardial fibres, coursing in all directions and even at right angles to each other. (c) Transverse view of both ventricles of child aged 14 months who died after pulmonary valvotomy. The left ventricle (LV) free wall is appreciably thicker than the right ventricle $(R V)$.
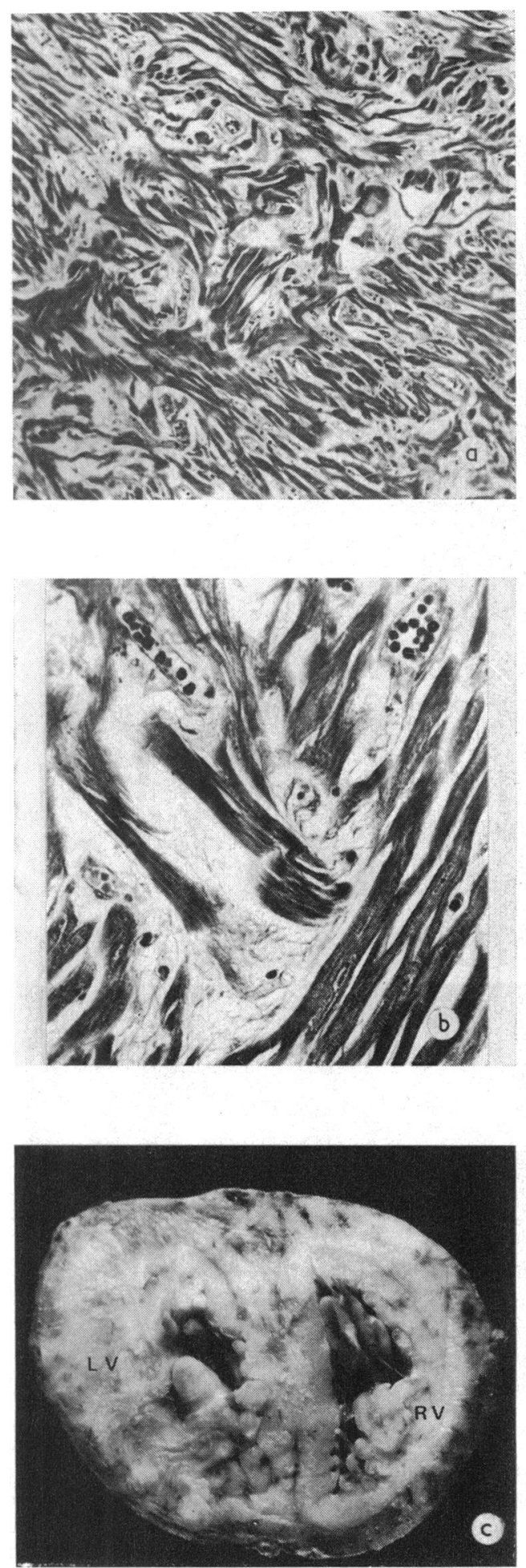
3 who survived, having had dyspnoea and radiological evidence of pulmonary oedema in the early postoperative days, since no structural lesion has been found to account for this course. One child 7 years later has developed electrocardiographic left ventricular hypertrophy, judged from voltage changes, and the other 2 children appear to be normal but have not been fully investigated. One boy had the stigmata of Noonan's syndrome (Noonan and Ehmke, 1963).

In an older group of 43 patients aged 3 to 20 years with right ventricular pressures 75 to $190 \mathrm{mmHg}$ $(10.0$ to $25.3 \mathrm{kPa})$, there were 8 with obvious features suggesting left-sided cardiovascular involvement (Table 3 ). The child in whom an aortic biopsy showed 'higgledy-piggledy' arteriopathy (Fig.
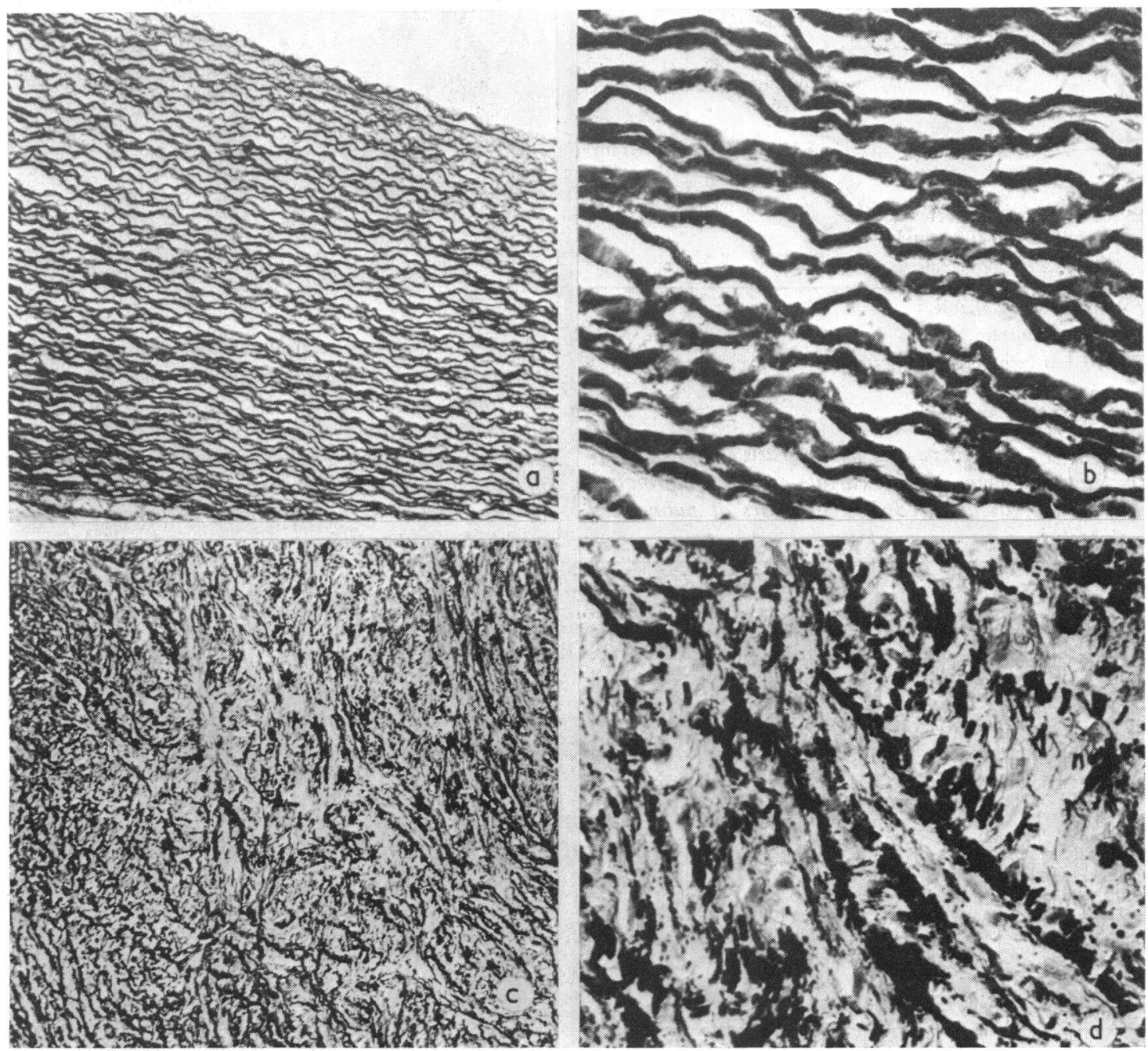

FIG. 4 Sections from the normal ascending aorta of a 5-month-old child who died from gastrointestinal disease in comparison with the aorta from a 6-month-old child who died with severe pulmonary valve stenosis. $(a)(\times 9)$ and $(b)(\times 35)$ from the normal child showing ordered parallel elastic fibres. $(c)(\times 9)$ and $(d)(\times 35)$ from the baby with pulmonary valve stenosis showing disorganization of elastic fibres with breaking up of elastic laminae which follow the smooth muscle components, typical appearances given the name 'higgledy-piggledy' disorder. Sections stained by Gomori's stain for elastic tissue. 
6) had rubella syndrome which was not present in the other patients. At the time of pulmonary valvotomy 5 years earlier, the pulmonary artery pressure was $45 / 22 \mathrm{mmHg}(6.0 / 2.9 \mathrm{kPa})$ despite a gradient of

TABLE 2 Postoperative findings suggesting diffuse cardiovascular involvement in 10 children aged 7 to 30 months who had pulmonary valvotomy

Low cardiac output state

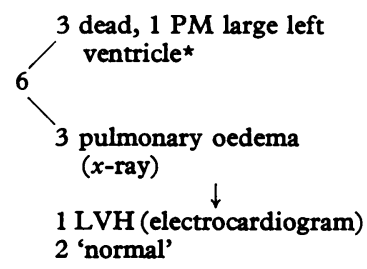

Systemic systolic hypertension 2

BP 140 to $160 / 100 \mathrm{mmHg}$

$(18.6$ to $21.3 / 13.3 \mathrm{kPa})$

*No histology available.

$\mathbf{P M}=$ post mortem.

LVH= Left ventricular hypertrophy.

TABLE 3 Operative and postoperative clinical findings suggesting more diffuse cardiovascular abnormality in 8 of 43 patients aged 3 to 20 years at pulmonary valvotomy

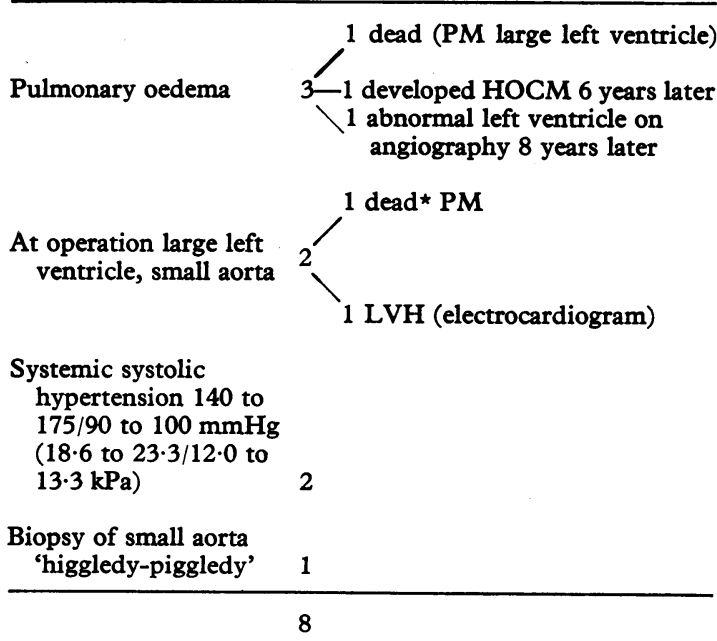

*In our opinion preliminary histology of small aorta showed classical 'higgledy-piggledy' and left ventricle was large. No further histology available.

$\mathbf{P M}=$ Post mortem.

HOCM $=$ Hypertrophic obstructive cardiomyopathy proven at operation.

$\mathbf{L V H}=$ Left ventricular hypertrophy.
$160 \mathrm{mmHg}(21.3 \mathrm{kPa})$ across the narrow pulmonary valve. He then developed severe left ventricular hypertrophy and was operated on for what was found to be mild tricuspid aortic valve stenosis

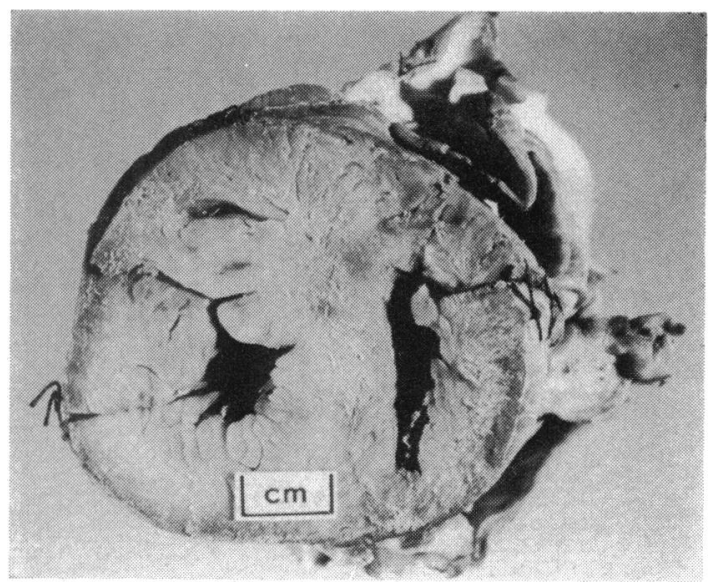

FIG. 5 Transverse section through the heart looked at from above, from an 11-month-old child reported in the clinical series (Table 2) who died 2 days after pulmonary valvotomy having been in a persistent low cardiac output state. The left ventricle marked with a cm marker is obviously abnormally thickened as is the right ventricle. Streaks of fibrous tissue were evident on naked eye inspection.

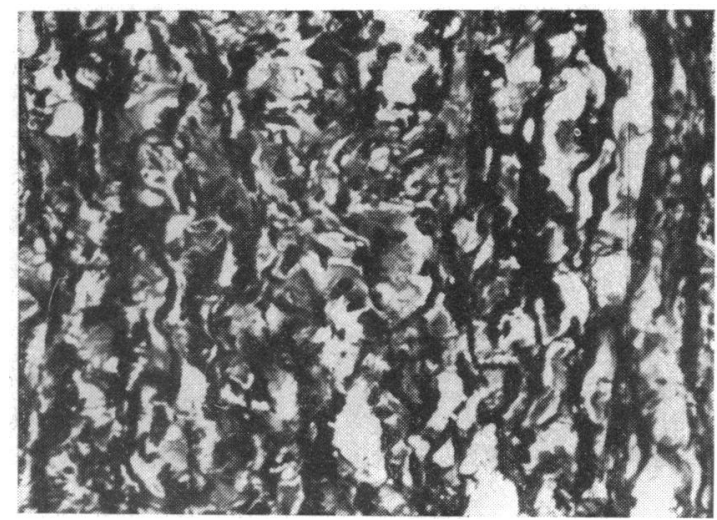

FIG. 6 Section from biopsy of ascending aorta in boy aged 10 years with rubella syndrome who had severe pulmonary valve stenosis. There are 'higgledypiggledy' changes with typical malalignment of elastic elements-stained with Gomori's aldehyde fuchsin $(\times 25)$. 
associated with severe diffuse subvalvar hypertrophy. Two of the other 7 patients with unusual left-sided problems after valvotomy had raised pulmonary artery pressures 30 to $35 \mathrm{mmHg}$ (4.0 to $4.7 \mathrm{kPa}$ ) before operation and none of the 7 had any structural disorder to account for the postoperative course. All these 8 patients had the thick tricuspid pulmonary valve stenosis already described, which was readily recognizable on right ventricular angiocardiography (Fig. 7).

In 1 of 3 patients, who developed postoperative dyspnoea and pulmonary oedema with left and right pleural effusions, the condition persisted for 2 months (Fig. 8). Investigation of this girl 8 years

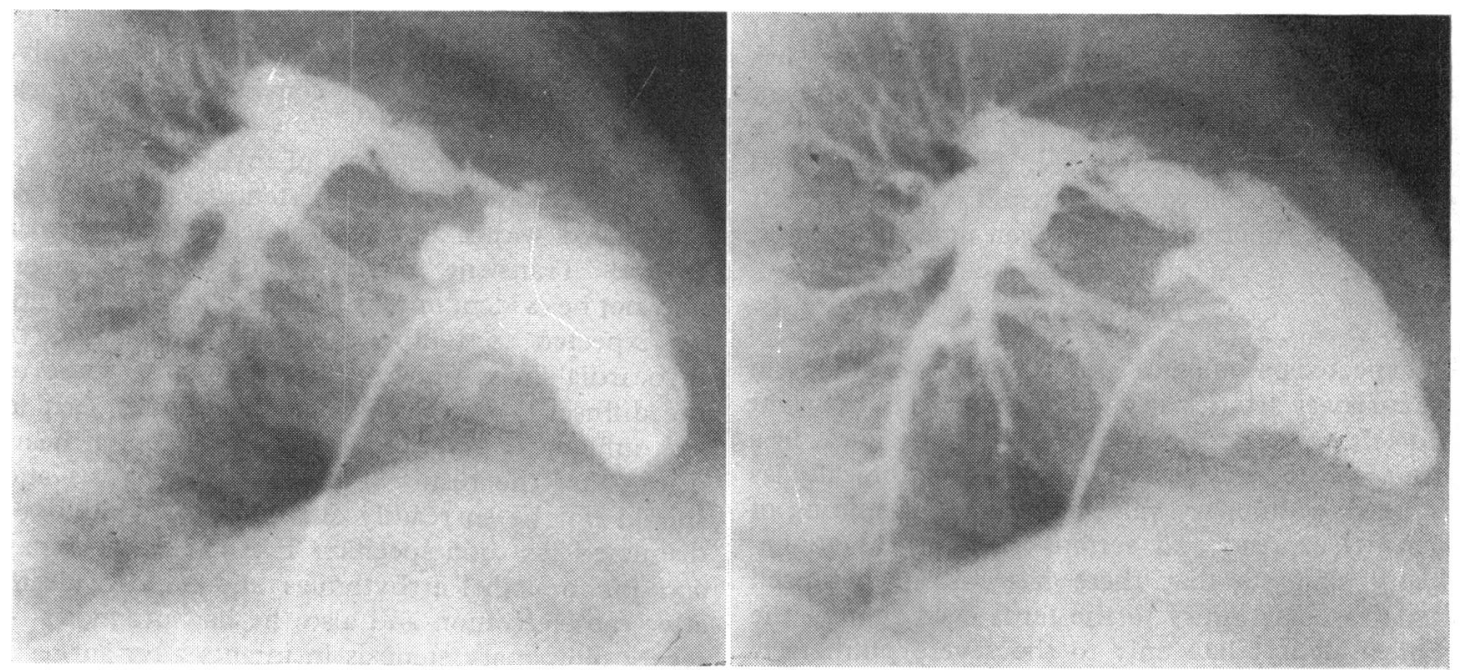

FIG. 7 Right ventricular angiocardiograms (lateral view) from a 26-month-old child with the thick tricuspid lumpy valve. Though domed in systole, the cusps are obviously thick and make up an irregular thickened filling defect in diastole (b). This is the type of pulmonary valve stenosis found in all those with clinical evidence of more diffuse cardiovascular involvement.

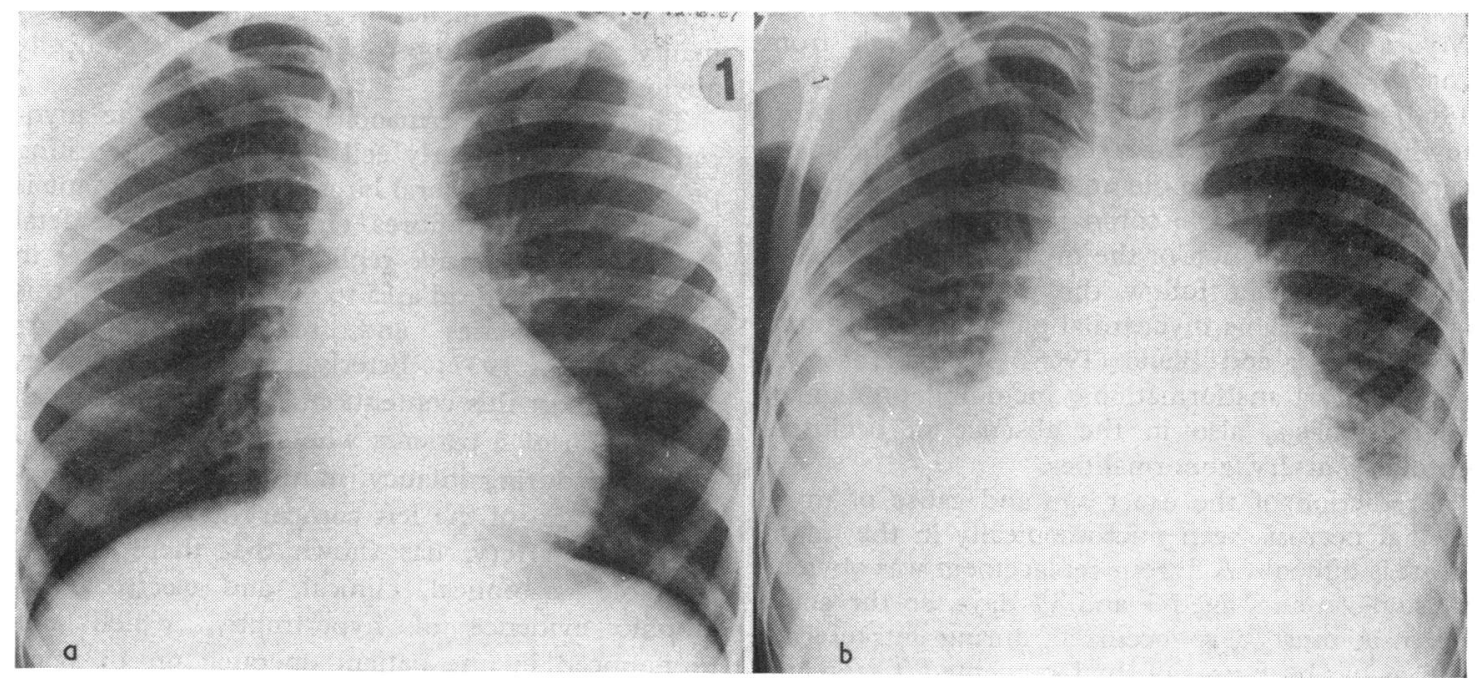

FIG. 8 Chest $\mathrm{x}$-ray films from a girl aged 11 years with severe pulmonary valve stenosis and resting right ventricular pressure of $180 \mathrm{mmHg}(23.9 \mathrm{kPa}):(a)$ before operation, (b) three weeks after operation, showing bilateral pleural effusions and pulmonary oedema which continued for 10 weeks. 
later showed abnormal thickening of the left ventricle with an $A$ wave of $17 \mathrm{mmHg}(2.3 \mathrm{kPa})$. The other child who survived a difficult postoperative period developed clinical features of obstructive cardiomyopathy which was confirmed by catheterization and resection of subaortic muscle 6 years after the pulmonary valvotomy.

Three of the 8 patients had Noonan's syndrome and the other 5 were somatically normal.

Incomplete survey of the other 35 patients has shown 3 patients with electrocardiographic signs of left ventricular hypertrophy and a further 5 have raised systemic blood pressure. These patients had the characteristic thick tricuspid valve but left ventricular function has not been investigated yet.

\section{Discussion}

Unexpected pathological abnormalities were found in the coronary arteries, in the myocardium of the right and left ventricles, and in the ascending aorta in a group of 25 patients who presented clinically as 'isolated' pulmonary valve stenosis. The finding of such widespread and serious pathological abnormality suggests that there has been generalized cardiovascular injury to the fetus even though the clinical signs relate only to the severe pulmonary valve stenosis.

Since widespread or severe coronary pathology was found in 17 of 25 specimens with 'isolated' pulmonary valve stenosis, and 12 of them had evidence of myocardial necrosis and focal fibrosis, a possible relation to each other must be considered. Myocardial necrosis in infancy can result from coronary occlusion as in adults (Gault and Usher, 1960) but it is unlikely to be a direct cause and effect in these patients because the severity of the myocardial changes, both old and recent, was unrelated to the severity of the coronary obstructive lesions, and the distribution of the myocardial fibrosis and necrosis did not follow the patterns of arterial supply. The same myocardial pathology was found by Franciosi and Blanc (1968) in different congenital heart malformations, including pulmonary valve stenosis, also in the absence of occlusive coronary artery abnormalities.

Prediction of the exact age and cause of myocardial necrosis seen microscopically in the infant heart is difficult. A fibrous replacement was obvious in some infants aged 1 and 17 days, so the acute necrosis must have occurred during intrauterine life, as has been previously documented (Clapp and Naeye, 1961).

Areas of recent necrosis were also found in older infants, some of whom had not had operation, suggesting that some new myocardial necrosis had occurred after birth. Berry (1967) reported similar myocardial damage in infants dying from a variety of conditions associated with anaemia, hypoxia, and hypotension. Thus, myocardial cell death may result in infants and children from disturbed cellular metabolism from several causes, just as Harris (1975) has postulated in adult myocardial infarction. Whatever the cause of the fetal injury or the pathogenesis of the cell death after birth, it appears to be common in severe isolated pulmonary valve stenosis and should be considered in clinical management.

The signs and symptoms of myocardial necrosis in infants with pulmonary stenosis may not be recognized within the general features of cardiac failure. Transient electrocardiographic changes may not be evident or may be missed and should not be expected to follow patterns characteristic of myocardial infarction in adults. Possibly some of the diffuse $T$ wave changes so frequently seen in sick infants with various types of congenital heart disease are the result of myocardial necrosis and should not be so readily attributed to digitalis or dismissed as non-specific. Fresh necrosis may account for lethal arrhythmias and sudden death after catheterization and also the bad prognosis of severe pulmonary stenosis in infants after surgery. Persistent abnormal ventricular function after good surgical relief of obstruction may possibly be the result of old healed necrosis in both ventricles. Studies on the left ventricle after pulmonary valvotomy in the clinical series are inadequate to elucidate the frequency of left ventricular abnormality except in those where it was clinically obvious.

The extent of permanent damage to the myocardium by such early cell death is an interesting problem. The collateral circulation in the infant is plentiful, and myofibres retain part of the fetal capacity for reparative replication. The mitoses in cells around the dead area so frequently seen in this pathological series and reported by others (MacMahon, 1937; Esterly and Oppenheimer, 1966) support this contention. Long-term personal observation of 3 patients who survived myocardial infarction during infancy, in association with anomalous origin of the left coronary artery from the pulmonary artery, has shown that there is considerable anatomical, clinical, and electrocardiographic evidence of hypertrophy, which was pronounced in one patient operated on 13 years later (Somerville and Ross, 1970).

Although pathological observations in the clinical series are scant and in the few who died there was no histological material available, there were at least 
2 in whom the left ventricle was large and fibrotic at necropsy, in addition to the clinical features summarized in Tables 2 and 3. In our opinion, preliminary histological investigation of the ascending aorta showed 'higgledy-piggledy' arteriopathy in the patient whose left ventricle was noted to be large at surgery, but further histological material was not available to us. Retrospective review of the opacified left ventricle seen on the late films from the right ventricular injection showed the left ventricle to be large and to contract abnormally and the small ascending aorta did not show the usual changes in size with diastole and systole. It may be that this left ventricular hypertrophy as also observed by Harinck (1974) in some late survivors with pulmonary stenosis may be a reactive development to earlier necrosis. Similarly dyspnoea, pulmonary oedema, and pulmonary effusion appearing after operation both in infants and occasionally in older patients may be secondary to this left ventricular disease.

Perhaps the most intriguing pathological finding in the myocardium is the appearance of disarray and malalignment of myocardial fibres referred to as 'myocardial dysplasia'. This may be another form of healing after fetal myocardial cell death or injury. It is likely that the myocardium, like other body tissues such as skin, has only a few limited ways of healing and this is one of them that can occur in the fetus or infant. On morphological grounds these appearances bear a resemblance to hypertrophic cardiomyopathy or asymmetrical septal hypertrophy (HOCM or ASH) (Ferrans, Morrow, and Roberts, 1972; Henry, Clark, and Epstein, 1973; Alexander and Gobel, 1974). The pathological evidence indicates that 'dysplasia' may involve extensive areas of both ventricles in some patients with pulmonary valve stenosis. Whether these patients would have developed later the classical clinical features of hypertrophic cardiomyopathy or just simple ventricular hypertrophy is unknown. Whether congenitally dysplastic myocardial fibres contract in an abnormal fashion and lead to further hypertrophy with necrosis and more fibrosis to give rise to the more classical histological features of hypertrophic cardiomyopathy or asymmetrical septal hypertrophy is also speculative. It may be relevant that the same sort of thick lumpy tricuspid pulmonary valve stenosis is the type found in Noonan's syndrome (Rainer-Pope et al., 1964) as also in hypertrophic cardiomyopathy (Ehlers et al., 1972) without added structural congenital heart disease. Furthermore, the frequent finding of an unusual electrical axis, either left anterior hemiblock or an unexpected deep $S$ in lead II of the electrocardiogram in Noonan type patients presenting with 'lone, pulmonary valve stenosis, may also be related to the presence of septal or left ventricular muscle disease or fibrosis.

In these patients, as in other congenital syndromes associated with pulmonary valve stenosis, there is evidence because of the presence of the other extracardiac manifestations that the congenital disease is not confined to the pulmonary valve.

It is also considered that the microscopical abnormality of disordered muscular and elastic fibres referred to as 'higgledy-piggledy' arteriopathy in the aorta should be regarded as a healing response to fetal injury and has been found in other examples of probable fetal damage. For instance, it was pronounced in a group of children presenting with systemic hypertension and found at necropsy to have increased heart weights for no apparent structural cause (Beçu and Gallo, 1974), in familial arteriopathy (McDonald, Gerlis, and Somerville, 1969), in supra-aortic stenosis of various cases (Somerville and Beçu, 1976), and was also obvious in the ascending aorta of the child in the clinical series with rubella syndrome. With the exception of the family whose great arteries looked like 'macaroni', the aorta looked macroscopically normal in all the patients, and the 'higgledy-piggledy' nature of elastic and muscle fibres was a histological finding. Such a disorder of major conducting arteries must induce impedance to pulse wave transmission, and perhaps this may be the cause of the raised systolic blood pressure noted in some survivors of pulmonary valvotomy as well as those known congenital syndromes with aortopathy and arteriopathy already referred to.

From these pathological findings it is suggested that a noxious fetal injury may be caused by one of several factors: rubella, chemical, metabolic, or genetic factors, and thus lead to myocardial necrosis and aortic pathology and in some instances may cause pulmonary valve stenosis. If the fetus survives, the myocardium may heal with fibrosis and compensatory hypertrophy or peculiar 'dysplasia' which might progress to the clinical picture of hypertrophic cardiomyopathy with or without 'higgledy-piggledy' aortopathy, another manifestation of healed disorder smooth muscular elements in the cardiovascular system.

Such theories if true may explain some of the associations of hypertrophic obstructive cardiomyopathy with other congenital heart abnormalities (Somerville and McDonald, 1968), and with strange congenital syndromes involving other organs outside the cardiovascular system, with or without pulmonary valve stenosis. All the clinical group with obvious evidence of left ventricular ab- 
normality had the thick tricuspid pulmonary valves, as described in the pathological series. However, serious myocardial and aortic changes were seen also in 4 of the 6 patients with thin tricuspid pulmonary valves. The pathological data are not extensive enough to show a significant difference between those with thin and thick valves. It is also likely that in the clinical series there is an underestimate of the problem because few have died, clinical observation has only recognized the very severely affected, and unawareness of the possibility of the diffuse nature of the disease has prevented recognition in both clinical and pathological observations.

In conclusion, it is suggested that 'isolated' pulmonary valve stenosis often may be a misnomer, particularly in patients presenting with angiographic evidence of thick lumpy pulmonary valves subsequently found to be tricuspid, in infants with severe obstruction, and in children who show also stigmata of diffuse congenital abnormality outside the cardiovascular system. The tricuspid primordia of the pulmonary valve in this group suggests the possibility that the fetal injury may have occurred later in fetal growth and not necessarily in the early weeks of cardiac development. How often patients presenting with a thin domed pulmonary valve or a bicuspid pulmonary valve have truly 'isolated' pulmonary stenosis is as yet unknown.

We believe these concepts may apply to other apparently 'isolated' or simple congenital heart abnormalities and that some including pulmonary valve stenosis should be regarded as 'congenital cardiovascular disease'.

We acknowledge gratefully the constructive criticism of Dr. G. Farrer-Brown, Bland Sutton Institute of Pathology, Middlesex Hospital Medical School. Professor Reginald Hudson kindly allowed us to photograph the specimen illustrated in Fig. 5. We thank Mr. Donald Ross for permitting publication of his observations at operation, and $\mathrm{Dr}$. Richard Emanuel for allowing us to examine his patient quoted in Table 3 and giving us access to the clinical information.

\section{References}

Alexander, C. S., and Gobel, F. L. (1974). Diagnosis of idiopathic hypertrophic subaortic stenosis by right ventricular septal biopsy. American fournal of Cardiology, 34, 142.

Beçu, L., and Gallo, A. (1974). Arteriosclerosis in infancy. European fournal of Cardiology, 1, 508.

Berry, C. L. (1967). Myocardial ischaemia in infancy and childhood. Fournal of Clinical Pathology, 20, 38.
Clapp, J. F., and Naeye, R. L. (1961). Intra-uterine myocardial infarction. Fournal of the American Medical Association, 178, 1039.

Ehlers, K. H., Engle, M. A., Levin, A. R., and Deely, W. J. (1972). Eccentric ventricular hypertrophy in familial and sporadic instances of $46 \mathrm{XX}, \mathrm{XY}$ Turner phenotype. Circulation, 45, 639.

Esterly, J. R., and Oppenheimer, E. H. (1966). Some aspects of cardiac pathology in infancy and childhood. I. Neonatal myocardial necrosis. Bulletin of the fohns Hopkins Hospital, $119,191$.

Ferrans, V. J., Morrow, A. G., and Roberts, W. C. (1972). Myocardial ultrastructure in idiopathic hypertrophic subaortic stenosis. A study of operatively excised left ventricular outflow tract muscle in 14 patients. Circulation, 45, 769 .

Franciosi, R. A., and Blanc, W. A. (1968). Myocardial infarcts in infants and children. I. A necropsy study in congenital heart disease. Fournal of Pediatrics, 73, 309.

Gault, M. H., and Usher, R. (1960), Coronary thrombosis with myocardial infarction in a new-born infant: clinical, electrocardiographic and post-mortem findings. New England Fournal of Medicine, 263, 379.

Harinck, E. (1974). Interaction of the right and left side of the heart and the post-natal changes thereof. M.D. Thesis, Leiden, pp. 82-96.

Harris, P. (1975). A theory concerning the course of events in angina and myocardial infarction. European fournal of Cardiology, 3, 157.

Henry, W. L., Clark, C. E., and Epstein, S. E. (1973). Asymmetric septal hypertrophy (ASH): the unifying link in the IHSS disease spectrum. Circulation, 47, 827.

McDonald, A. H., Gerlis, L. M., and Somerville, Jane (1969). Familial arteriopathy with associated pulmonary and systemic arterial stenoses. British Heart fournal, 31, 375.

MacMahon, H. E. (1937). Hyperplasia and regeneration of the myocardium in infants and in children. American fournal of Pathology, 13, 845.

Neufeld, H. N. (1974). Studies of the coronary arteries in children and their relevance to coronary heart disease. European fournal of Cardiology, 1, 479.

Noonan, J. A., and Ehmke, D. A. (1963). Associated noncardiac malformations in children with congenital heart disease. Fournal of Pediatrics, 63, 468.

Rainer-Pope, C. R., Cunningham, R. D., Nadas, A. S., and Crigler, J. F., Jr. (1964). Cardiovascular malformations in Turner's Syndrome. Pediatrics, 33, 919.

Shorter Oxford English Dictionary (1944). 3rd ed. p. 901. Clarendon Press, Oxford.

Somerville, J., and Beçu, L. (1976). Aortic wall pathology in supra-aortic stenosis and isolated congenital cardiac malformation. 7th Congress of European Society of Cardiology, Amsterdam.

Somerville, J., and McDonald, L. (1968). Congenital anomalies in the heart with hypertrophic cardiomyopathy. British Heart fournal, 30, 713.

Somerville, Jane, and Ross, D. N. (1970). Left coronary artery from the pulmonary artery: physiological considerations of surgical correction. Thorax, 25, 207.

Requests for reprints to Dr. Jane Somerville, Cardiothoracic Institute, 2 Beaumont Street, London W1N 2DX. 